\title{
Polarimetric SAR Image Classification with High Frequency Component Derived from Wavelet Multi Resolution Analysis: MRA
}

\author{
Maximum Curvature of Trajectory of Back Scattering Cross Section Converted from Eigen \\ Space of Ellipticity and Orientation Angle Space of Polarization Signature
}

\author{
Kohei Arai \\ Department.of Information Science, \\ Graduate School of Science and Engineering \\ Saga University \\ Saga city, Japan
}

\begin{abstract}
A method for polarimetric Synthetic Aperture Radar: SAR image classification with high frequency component derived from wavelet Multi-Resolution Analysis: MRA is proposed. Although it is well known that polarization signature derived from fully polarized SAR data is useful for SAR image classifications, it is still unknown how to utilize the polarization signature in the image classification. High frequency component of the polarization signature calculated with the fully polarized SAR data is taken as one of the features utilizing in the classification into account. Thus improvement of classification performance is achieved for the proposed classification method of which such feature is included in the feature space for the Maximum Likelihood based classification method.
\end{abstract}

Keywords- polarimetric SAR; polarization signature; wavelet MRA.

\section{INTRODUCTION}

Radar polarimetry allows measurement the physical characteristics such as di-electric constant, slope of the ground cover targets as well as directionality of artificial objects by using scattering mechanism between electromagnetic (EM) wave and the targets [1],[2]. Polarimetric SAR image classification with the following three components of the polrimetric SAR data, (1) transmit Electro-magnetic wave with Horizontal Polarization(H-Pol) and receive the echo from the ground with $\mathrm{H}-\mathrm{Pol}(\mathrm{HH})$, (2) transmit Electro-magnetic wave with $\mathrm{H}-\mathrm{Pol}$ and receive the echo with Vertical Polarization(V-Pol)(HV) and (3) transmit Electro-magnetic wave with $\mathrm{V}$-Pol and receive the echo with $\mathrm{V}$-pol(VV) is widely available [3],[4]. On the other hand, the extraction of the scattering characteristics of the targets of interest by applying eigen value decomposition to the covariance matrix derived from the scattering matrix which is calculated from the three components are proposed [5]. Furthermore, the classification methods with the single / double /multiple, odd / even / diffuse, and odd / even / Bragg / multiple scattering components derived from the eigen value decomposition were proposed [6] while the classification methods with the sphere / deplane / helix, and sphere / Bragg / double of scattering components which are based on the spherical polarization which are derived from the scattering matrix were also proposed [7],[8].

Aforementioned proposed methods were reviewed [9]. Moreover, the classification method with the entropy $(\mathrm{H})$ which is defined with the sum of the first to third eigen values and the ratio of each eigen values, the anisotropy (A) which is defined as the ratio of sum and subtraction of the second and the third eigen values and $\operatorname{cosine} \alpha(\cos (\alpha))$ which is defined with the elements of the eigen vector corresponding to the first eigen value which is called coherency matrix(3 by 3 ) was proposed by E.Pottier [10].

The application of these methods to sea ice discrimination (such as thin ice (TI), smooth first year ice (SF), rough first year ice $(\mathrm{RF})$ and open water $(\mathrm{OW})$ ) with the polarimetric SAR were attempted by using $H, A$, and $\cos (\alpha)$ [11]. Classification performance, however, were not satisfactory (20-40\% of classification errors were occurred for the classification of sea ice into four classes, ridged, compressed, new forming and smooth surface due to the fact that scattering mechanism based features were not used effectively. Meanwhile polarimentirc SAR image classification with polarization signature which are derived from Stokes or Muller or scattering matrix is widely available [12]. Polarization signature represents the scattering mechanism, in particular, surface roughness of the targets in concern.

One of the problems on the classification with polarization signature is classification performance. The method for effective utilization of polarization signature is still unclear to improve classification performance. The method proposed here is for extraction of effective information from the polarization signature by transforming the polarization signature onto an eigen space (eigen value decomposition). As the results from the eigen value decomposition which corresponds to the largest eigen value, a trajectory can be drawn. The trajectory represents the scattering mechanism in concern so that the largest curvature of the trajectory represents the most effective representatives of the scattering mechanism of the target of interest [13]. This is the theoretical 
background to propose the utilization of maximum curvature of the trajectory in an eigen space which is derived from the polarization signature to the sea ice classification.

Firstly, the proposed method is introduced with a theoretical background followed by experimental data and the results from the experimental are described together with the results from a comparative study between the proposed method and the existing conventional methods. Finally, concluding and remakes with some discussions is followed.

\section{PROPOSED METHOD}

\section{A. Polarization Signature}

Received echo signal $\left(E_{h}, E_{v}\right)^{T}{ }_{r e c}$ of polarimetric SAR is expressed as follows,

$$
\begin{aligned}
& \left(\begin{array}{l}
E_{h} \\
E_{v}
\end{array}\right)_{r e c}=\frac{e^{-j k R}}{k R}\left(\begin{array}{ll}
S_{h h} & S_{h v} \\
S_{v h} & S_{v v}
\end{array}\right)\left(\begin{array}{l}
E_{h} \\
E_{v}
\end{array}\right)_{i l l} \\
& S=\left(\begin{array}{ll}
S_{h h} & S_{h v} \\
S_{v h} & S_{v v}
\end{array}\right)
\end{aligned}
$$

where $S$ is a scattering matrix composed with $\mathrm{HH}, \mathrm{HV}, \mathrm{VH}$ and VV components of $S_{h h}, S_{h v}, S_{v h}$ and $S_{v v},\left(E_{h}, E_{v}\right)^{T}$ ill denotes incident Electro-magnetic wave, $k$ is wave number of the incident Electro-magnetic wave, $R$ denotes range, respectively. From these elements of $\mathrm{S}$, Muller matrix is calculated while Stokes vector at the receiver $J_{r}$ is calculated with $\left(E_{h}, E_{v}\right)^{T}$ rec and also Stokes vector at the transmitter $J_{t}$ is calculated with $\left(E_{h}, E_{v}\right)^{T}{ }_{i l l}$ thus the polarization signature $\sigma^{0}$ is represented by the following equation,

$$
\begin{aligned}
& \sigma^{0}=K J_{r}^{T}\left\langle M_{s}\right\rangle J_{t}=\sigma^{0}\left(\chi_{r}, \phi_{r}, \chi_{t}, \phi_{t}\right) \\
& K: \text { const. }
\end{aligned}
$$

where the $\chi$ and $\varphi$ denote the ellipticity angle and the orientation angle of the orientation of the electric field vector, respectively of which the polarization is described with the two parameters. The polarization signature describes the scattering coefficient as a function of any assumed transmit and receive antenna polarization and allows measure the variation of the scattering coefficient with polarization so that the different targets show the different polarization signature as shown in Fig.1.

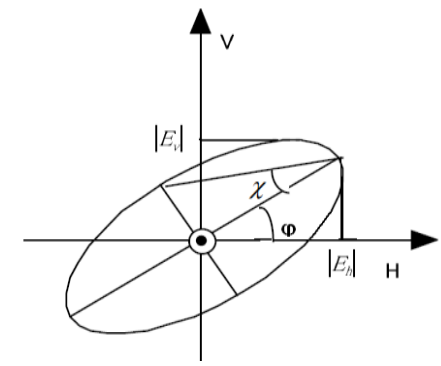

Figure 1. Definition of polarization signature

The method for effective utilization of polarization signature is still unclear to improve classification performance. follows,

Let $\mathrm{X}$ be matrix with three scattering components as

$$
\begin{gathered}
X=\left[S_{h h} S_{h v} S_{v v}\right]^{T} \quad C=<X X^{* T}> \\
C=\lambda_{1} K_{1}\left(K_{1}^{*}\right)^{T}+\lambda_{2} K_{2}\left(K_{2}^{*}\right)^{T}+\lambda_{3} K_{3}\left(K_{3}^{*}\right)^{T}
\end{gathered}
$$

where $C$ denotes covariance matrix of $X$ and $\lambda_{1}, \lambda_{2}, \lambda_{3}$ are eigen values of $C$. These are corresponding to odd times scattering, even times scattering and diffuse scattering, respectively. Also * and $\mathrm{T}$ denotes complex conjugate and transpose, respectively. Thus

$\frac{\lambda_{i}}{\lambda_{1}+\lambda_{2}+\lambda_{3}} \quad(i=1,2,3)$

is called a contribution factors. If the equation (4) are formulated,

$$
\begin{gathered}
S_{R R}=\frac{1}{2}\left(S_{h h}-S_{v v}+j 2 S_{h v}\right) \\
S_{L L}=\frac{1}{2}\left(S_{h h}-S_{v v}-j 2 S_{h v}\right) \\
S_{L R}=\frac{1}{2}\left(S_{h h}+S_{v v}\right)
\end{gathered}
$$

then spherical, di-plane and helix components are defined as follows,

$K_{s}=\left|S_{L R}\right| \quad K_{d}=\left|S_{L L}\right| \quad K_{h}=\left|S_{R R}\right|-\left|S_{L L}\right|$ for $\left|S_{R R}\right|>\left|S_{L L}\right|$

Thus contribution factor for each is defined as follows,

$\frac{K_{i}}{K_{s}+K_{d}+K_{h}} \quad(i=s, d, h)$

Polarization signature represents the polarization feature of the ground cover target, obviously.

\section{B. Proposed Method}

Classification method proposed here is to use wavelet frequency components as features for classification. In particular, high frequency components derived from wavelet Multi Resolution Analysis: MRA is one of spatial feature of the target class. One dimensional wavelet transformation is expressed with the equation (7).

$F=C n f$

where $F, f$ denotes wavelet frequency component and radar echo signal as a function of time. $C n$ denotes wavelet transformation matrix which is expressed as a bi-orthogonal function based on base functions. $C n$ can be determined with a reference to the appendix. Therefore, $\mathrm{CnCn}^{t}=I$. Then $f$ is converted to $F_{1}=\left(L_{1}, H_{1}\right), F_{2}=C n L_{1}=\left(L_{2}, H_{2}\right), F_{3}=C n L_{2}=\left(L_{3}, H_{3}\right)$, and $F_{m}=C n L_{m-1}=\left(L_{m}, H_{m}\right)$. Also $f$ is reconstructed as

$C n^{-1} F_{m}=C n^{-1}\left(L_{m}, H_{m}\right)=L_{m-1}, \ldots \ldots, C n^{-1} F_{2}=L_{1}, C n^{-1} F_{l}=f$. The suffix of 1 to $\mathrm{m}$ is called "level". Level $\mathrm{m}$ implies that wavelet transformation is applied $\mathrm{m}$ times. MRA ensure that the original signal can be reconstructed with the wavelet coefficients or frequency components of level $\mathrm{m}$. The frequency components derived from MRA are corresponding to the level $\mathrm{m}$. Therefore, MRA does work as filter bank. 
There are some based functions such as Haar, Daubechies ${ }^{1}$, etc. Through the experiment with preliminary simulation of radar echo data, Daubechies base function is selected. Daubechies base function is one of bi-orthogonal functions. MRA is applied to the polarization signature then the extracted high frequency component is added to the typical features of received polarization power signals, $\mathrm{HH}, \mathrm{HV}$, and $\mathrm{VV}$ components where $\mathrm{HH}$ denotes the horizontal polarization of electro-magnetic wave is transmitted then the returned echo signal is received in horizontal polarization. Thus spatial feature of the polarization of feature of the ground cover target is taken into account in the classifications.

Typically, three polarization power signals are acquired with polarimetric SAR system.

\section{Methods for Comparison}

The classification performance of the proposed method is compared to the Maximum Likelihood classification with only three components of the polarimetric SAR data of $\mathrm{HH}, \mathrm{HV}$, $\mathrm{VV}$, with the three components $+H+A+\cos (\alpha)$, with the three components $+C_{l}$, and with the three components + Odd + Even + Diffuse for discrimination among the following three classes, Urban, Vegetation, and Paddy field. Also classification performance is compared with the previously proposed classification method with information from the polarization signature by using eigen value decomposition of the polarization signature. As the results from the eigen value decomposition which corresponds to the largest eigen value, a trajectory can be drawn. The trajectory represents the scattering mechanism in concern so that the largest curvature $\left(C_{l}\right)$ of the trajectory represents the most effective representatives of the scattering mechanism of the target of interest. If the largest curvature is large, then the polarization signature is steeply while the largest curvature is small, then the polarization signature is calm. The proposed classification method is based on the well known maximum likelihood classification with the received signal of the three different polarizations, co-polarization ( $\mathrm{HH}$ and VV) and crosspolarization (HV) as well as the maximum curvature of the trajectory in the eigen space through the eigen value decomposition from the polarization signature.

\section{EXPERIMENTS}

\section{A. Data Used}

The PI-SAR (Polarimetric and Interferometric SAR) data of Tsukuba in Japan which was acquired by CRL (Communication Research Laboratory, current NICT: National Institute of Communication Technology) and NASDA (National Space Development Agency of Japan, current JAXA: Japan Aeronautics Exploration Agency) on 23

${ }^{1}$ Daubechies base function is defined as $\left\{\alpha_{\mathrm{k}}\right\}$ satisfying the following conditions,

$$
\begin{aligned}
& \phi(x)=\sum_{k} \alpha_{k} \sqrt{2} \phi(2 x-k) \\
& \beta_{k}=(-1)^{k} \alpha_{1-k} \\
& \psi(x)=\sum_{k} \beta_{k} \sqrt{2} \phi(2 x-k)
\end{aligned}
$$

Feb. 1999 was used. The major characteristics of the PI-SAR are in Table 1.

TABLE 1. MAJOR CHARACTERISTICS OF PI-SAR

\begin{tabular}{|l|l|}
\hline Instrument & NASDA/L-band SAR \\
\hline Center frequency & $1.27 \mathrm{GHz}$ \\
\hline Peak power & $3.5 \mathrm{~kW}$ \\
\hline Band width & $50 \mathrm{MHz}$ \\
\hline Antenna size & $1.6 \mathrm{~m} \mathrm{x} \mathrm{0.7m}$ \\
\hline Polarization & $\mathrm{HH} / \mathrm{HV} / \mathrm{VH} / \mathrm{VV}($ Full Pol.) \\
\hline Incident angle & $20-60 \mathrm{degrees}$ (Fixed) \\
\hline Swath width & $42.5 \mathrm{~km}$ \\
\hline Spatial resolution & $3 \mathrm{~m}$ \\
\hline Quantization bit & $8 \mathrm{bits}(\mathrm{I}$ and Q) \\
\hline
\end{tabular}

From the data of SSC (Single-look Slant-range Complex) of the data,

\section{B. Polarization Signature}

Fig.2 shows an example of SSC (Single-look Slant-range Complex) data (one pixel is corresponding to 3 by 3 meters)of Tuskuba city, Japan which is acquired on 30 September 1997. Blue, yellow, and green square shows the training areas of Paddy fields (After harvest), Urban areas, and Vegetated areas, respectively.

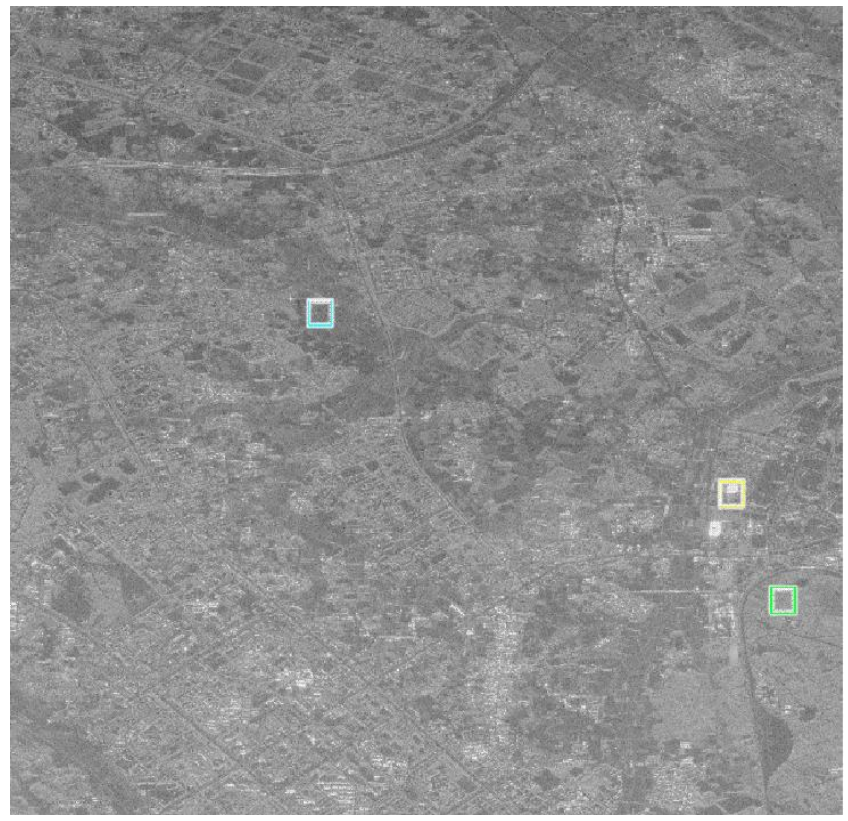

Figure 2. An example of SSC (Single-look Slant-range Complex) data of Tuskuba city, Japan which is acquired on 30 September 1997.

Using equation (1), polarization signature can be calculated by pixel by pixel because the parallel polarization, Co-pol. and cross polarization, Cross-Pol. are acquired by pixel by pixel. Fig.3 shows the calculated polarization signature for each class. The calculated polarization signatures are different each other. Therefore, it can be classified. The problem of the conventional classification method which uses the polarization signature and the returned echo signal powers, $\mathrm{HH}, \mathrm{HV}$, and $\mathrm{VV}$ is poor classification performance. The proposed method utilizes 
high wavelet frequency components other than these polarization signature and returned echo signal powers.

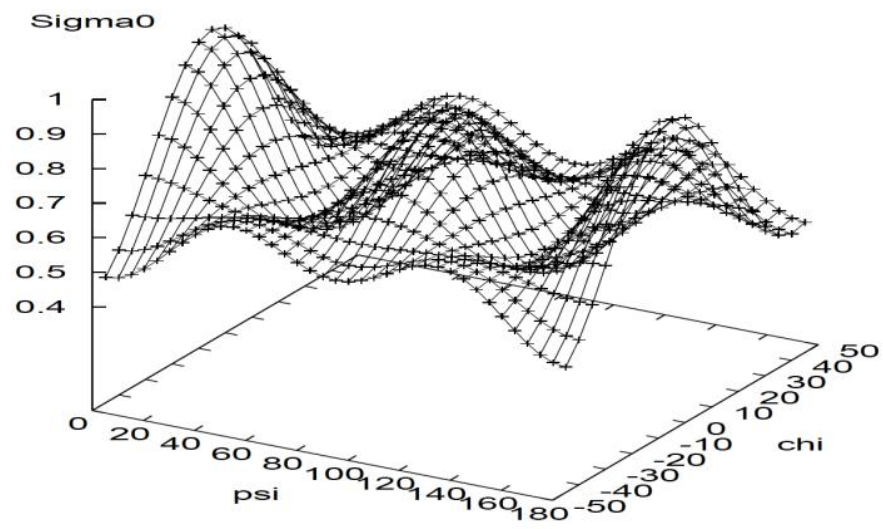

(a) Urban (Co-pol)

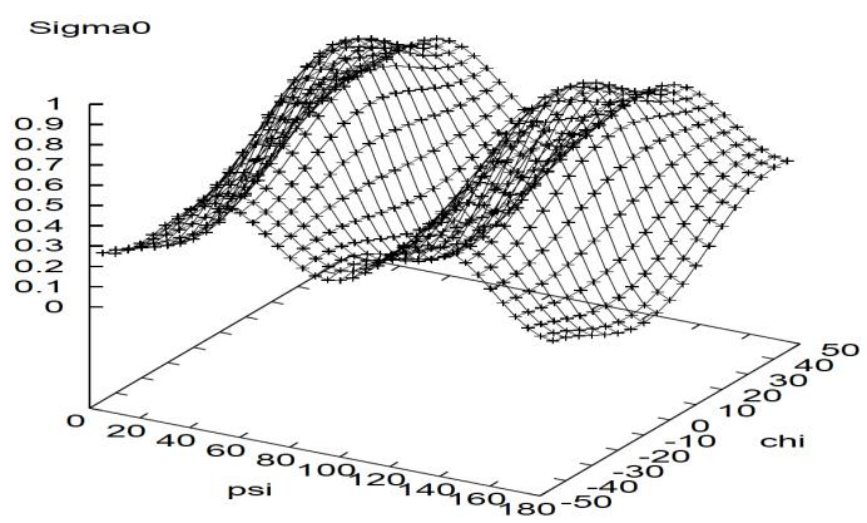

(b)Urban (Cross-pol)

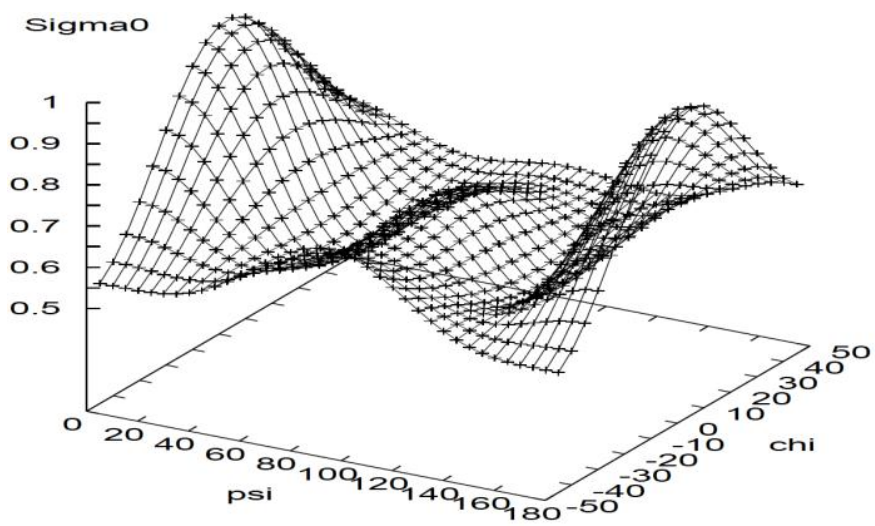

(c) Vegetation (Co-pol)

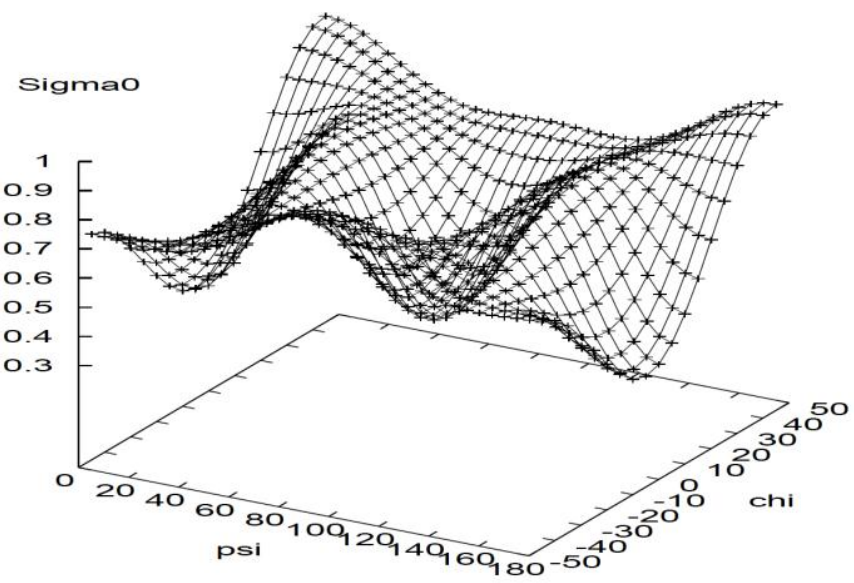

(d) Vegetation (Cross-pol)

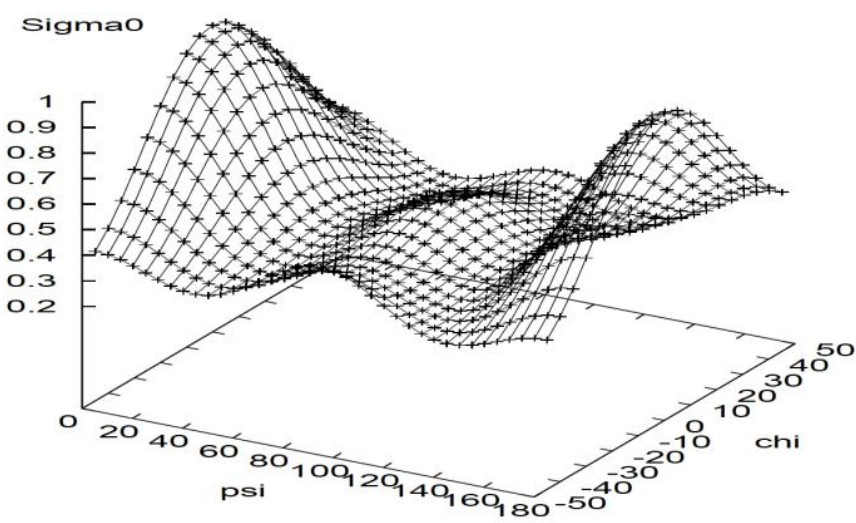

(e)Paddy (Co-pol)

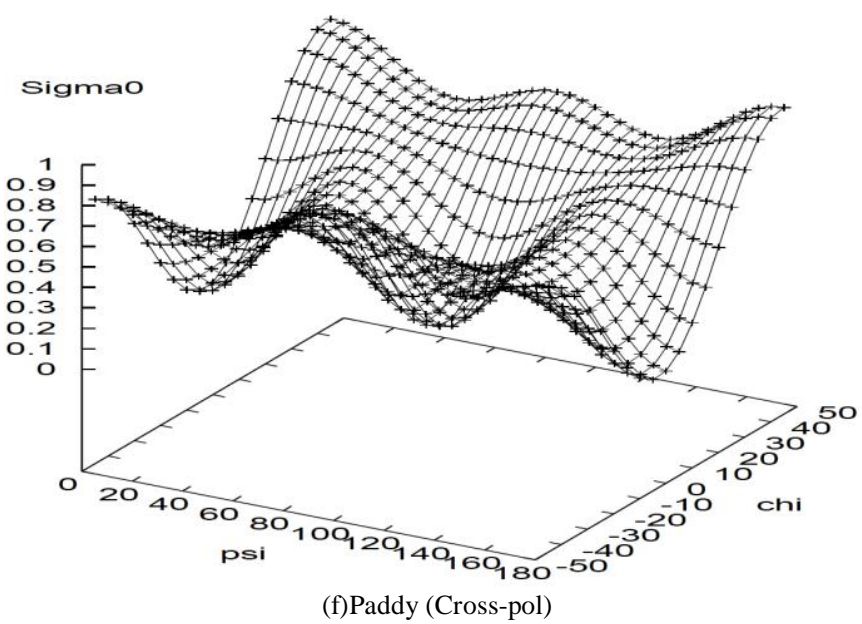

Figure 3. Examples of the calculated polarization signatures of the classes, Urban, Vegetation, and Paddy extracted from the 16 by 16 pixels of square areas which are corresponding to the designated classes. 


\section{Class Features}

Because the PI-SAR frequency is $1.27 \mathrm{GHz}$, the contribution of leaves of vegetation is very week (penetrated) while the contribution from the ground surface and the relatively large trees is major. Therefore, polarization signature of vegetated areas is similar to the paddy fields after harvest except surface flatness. On the other hand, HH return echo signal power and polarization signature for both urban areas and vegetated areas are similar. As the results from the aforementioned reasons, all these three classes are difficult to classify. It is confirmed that three components of return echo signal power, $\mathrm{HH}, \mathrm{HV}$, and $\mathrm{VV}$ utilized classification makes about $80 \%$ of classification performance.

In order to improve classification performance, the following feature is proposed by the author. That is maximum curvature of the trajectory in the eigen vector space which is converted from the polarization signature with arbitrary polarization angle response with 5 degrees intervals. The proposed feature does work for classification for the classes with different surface roughness, in particular. Although it is effective to improve classification performance, it is complicated and requires computation resources.

The proposed classification method is based on wavelet frequency components of the calculated polarization signature. Therefore not significant computation resources are not required and it is quite simple.

Fig.4 (a) and (b) shows examples of the high frequency component (HH4) of the polarization signature of image which is shown in Fig.3, and the maximum curvature of the trajectory in eigen vector space which is converted from the polarization signature image, respectively.

It is difficult to discriminate between urban and vegetation classes because the previously proposed maximum curvature of urban and vegetation is similar as shown in Fig.4 (b). On the other hand, the proposed feature of high frequency component $\mathrm{HH} 4$ of image shows clear difference between urban and vegetation classes. Therefore, HH4 frequency component which is derived from the level 4 of MRA output, HH4, HL4, LH4, and LL4 of the image which consists of 32 by 32 pixels with 5.6 degree step of the polarization angle of the each dimension of polarization signature is used for classification.

\section{Classification Performance}

Using training samples extracted from the training areas which are indicated in Fig.2, training performance is evaluated. A comparison is made for the following three Maximum Likelihood based classification method with (1) received three echo signal power, HH, HV, VV, with (2) three power and the previously proposed maximum curvature, and with (3) three power and the $\mathrm{HH} 4$ of each dimension of polarization signature.

Classification performance is evaluated with confusion matrix and the $\mathrm{k}$ statistics which is shown in the following equation (8),

$$
k=\frac{P(A)-P(E)}{1-P(E)}
$$

where $\mathrm{P}(\mathrm{A}), \mathrm{P}(\mathrm{E})$ denotes correct classification probability and classification error probability. Table 2, 3,4 shows the results.

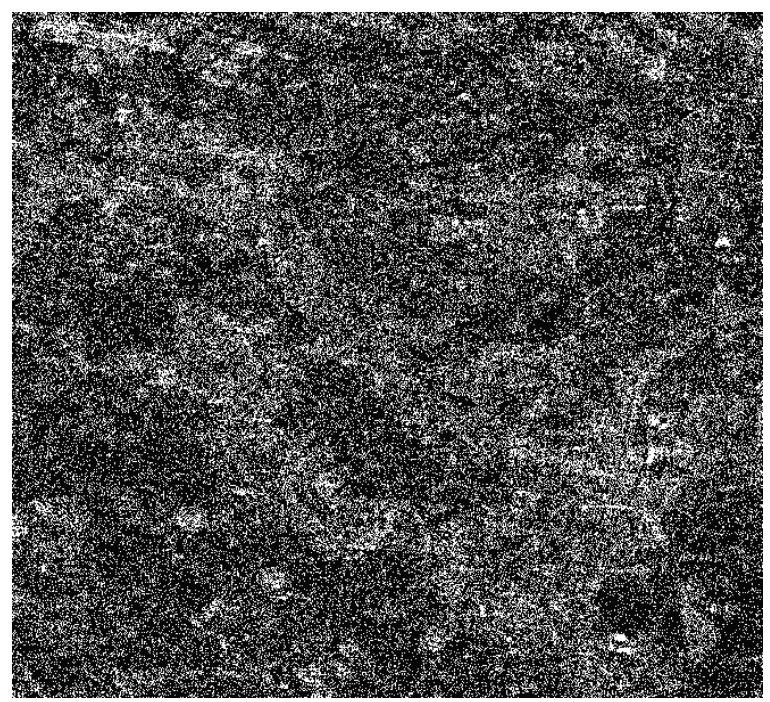

(a) Examples of the high frequency component of $\mathrm{HH} 4$ of the polarization signature of image which is shown in Fig.3

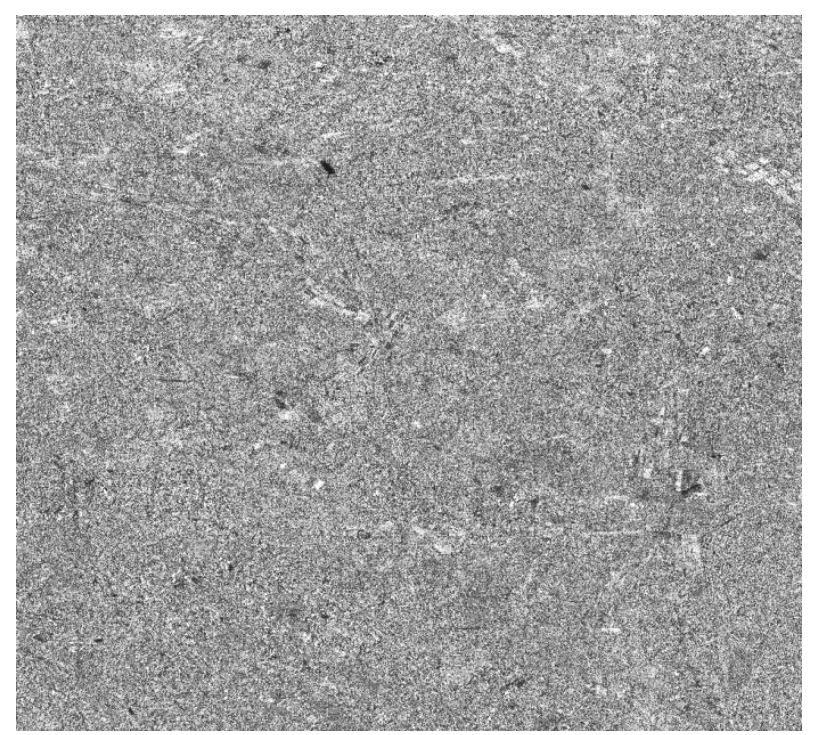

(b) The maximum curvature image of the trajectory in eigen vector space which is converted from the polarization signature image

Figure.4. The image of the features of the previously proposed maximum curvature of the trajectory in eigen space which is converted from the polarization signature and the high wavelet frequency component image of the polarization signature.

TABLE 2. THREE RECEIVED ECHO SIGNAL POWER ONLY ( $\mathrm{K}=89.05$ )

\begin{tabular}{|c|r|r|r|}
\hline & \multicolumn{1}{|c|}{ Urban } & Vegetation & \multicolumn{1}{c|}{ Paddy } \\
\hline Urban & 75.2 & 24.8 & 0.0 \\
\hline Vegetation & 2.4 & 97.6 & 0.0 \\
\hline Paddy & 0.8 & 1.6 & 97.6 \\
\hline
\end{tabular}


TABLE 3. THREE POWER AND THE MAXIMUM CURVATURE ( $\mathrm{K}=95.62)$

\begin{tabular}{|c|r|r|r|}
\hline & \multicolumn{1}{|c|}{ Urban } & Vegetation & \multicolumn{1}{c|}{ Paddy } \\
\hline Urban & 89.8 & 10.2 & 0.0 \\
\hline Vegetation & 1.2 & 98.8 & 0.0 \\
\hline Paddy & 0.4 & 0.8 & 98.8 \\
\hline
\end{tabular}

TABLE 4. THREE POWER AND HH4 (K=98.0)

\begin{tabular}{|c|r|r|r|}
\hline & \multicolumn{1}{|c|}{ Urban } & Vegetation & \multicolumn{1}{c|}{ Paddy } \\
\hline Urban & 94.9 & 5.1 & 0.0 \\
\hline Vegetation & 0.0 & 100.0 & 0.0 \\
\hline Paddy & 0.0 & 0.8 & 99.2 \\
\hline
\end{tabular}

As is shown in the previous section, it is difficult to discriminate between urban and vegetation classes if only three power of features are used. It is improved by adding the features of maximum curvature and $\mathrm{HH} 4$, remarkably. On the other hand, k-statistics shows that $7.38 \%$ of improvement is achieved by adding the maximum curvature and $10.05 \%$ improvement is achieved by adding HH4.

\section{CONCLUSION}

It is found that the proposed features of three received echo signal power, $\mathrm{HH}, \mathrm{HV}$, and $\mathrm{VV}$ as well as $\mathrm{HH} 4$ of high frequency component derived from MRA analysis with polarization signature are effective to improve classification performance for PI-SAR types of polarimetric SAR images. More than $10 \%$ of improvement of the classification performance is confirmed by adding $\mathrm{HH} 4$. The improvement depends on the level of MRA, because there is the most appropriate level depending on the frequency component of the polarization signature of the ground cover target.

The computational resource requirement for the previously proposed maximum curvature of the trajectory in eigen vector space which is converted from the polarization signature is twice much larger than that of the proposed MRA based feature utilized classification. Thus it may said that the proposed high frequency component of polarization signature based classification method is superior to the conventional classification method with three echo signal powers, and to the previously proposed maximum curvature based classification method in terms of classification performance and the required computation resources.

\section{REFERENCES}

[1] Zebker, H.A., vanZyl, J.J. Imaging radar polarimetry: A review. Proc. IEEE 79, 1583-1606, 1991.

[2] Ulaby, F.T., Elachi, C. Radar Polarimetry for Geoscience Applications. Artech House Inc., Dedham, 1990.

[3] Mott, M. Antennas for Radar Communications - A Polarimetric Approach. John Wiley, New York, 1992.

[4] Henderson, F.M., Lewis, A.J. Principles and applications of imaging radar, in: Manual of Remote Sensing, third ed. John Wiley, New York, 1998.

[5] Krogager, E. Analysis of the absolute and relative phase conditions of transforming matrices for the sinclair and covariance matrices in radar polarimetry. J. Opt. Soc. Am. E72B, 1A, 1993.

[6] Dong, Y. Imaging radar polarimeter from wave synthesis. J. Geophys. Res. 92 (81), 638-701, 1987.

[7] Zebker, H.A., et al., Imaging radar polarimeter from wave synthesis, J. Geophysical Research, Vol.92, No.81, pp.638-701, 1987.

[8] Krogager, E., Czyz, Z.H. Properties of the sphere, di-plane, helix decomposition, in: Proceedings, Third International Workshop on Radar Polarimetry, vol. 1, pp. 106-114, 1995.

[9] Cloude, S.R., Pottier, E. A review of target decomposition theorems in radar polarimetry. IEEE Trans. Geosci. Remote Sensing 34 (2), 498-518, 1995.

[10] Pottier, E., Lee, J.S. Unsupervised classification scheme of Polsar images based on the complex Wishart distribution and H/A/a polarimetric decomposition theorems, Proceedings of the Third European Conference on Synthetic Aperture Radar: EUSAR, 2000.

[11] Scheuchl, B., Hajinsek, I., Cumming, I.G., et al. Sea ice classification using multi-frequency polarimetric SAR data, in: Proceedings of the IGARSS'02, 2002.

[12] vanZyl, J.J., Zebker, H.A., Elachi, C. Imaging radar polarization signatures, theory and observation. Radio Sci. 22 (4), 529-543, 1987.

[13] Kohei Arai and J.Wang, Polarimetric SAR image classification with maximum curvature of the trajectory in eigen space domain on the polarization signature, Advances in Space Research, 39, 1, 149-154, 2007.

\section{AUTHORS PROFILE}

Kohei Arai received a PhD from Nihon University in 1982. He was subsequently appointed to the University of Tokyo, CCRS, and the Japan Aerospace Exploration Agency. He was appointed professor at Saga University in 1990. He is also an adjunct professor at the University of Arizona and is Vice Chairman of ICSU/COSPAR Commission A. 